\title{
Piezoelectric actuation of PZT thin film diaphragms at static and resonant conditions
}

\author{
P. Muralt, A. Kholkin, M. Kohli, and T. Maeder \\ Laboratoire de Céramique, EPFL, MXD Ecublens CH-1015 Lausanne, Switzerland. \\ Version of record: Sensors and Actuators A 53 (1-3), 398-404, 1996. \\ http://hdl.handle.net/10.1016/0924-4247(96)01139-9
}

\begin{abstract}
The piezoelectric response of silicon diaphragms covered with sputter-deposited $\mathrm{PbZr}_{0.45} \mathrm{Ti}_{0.55} \mathrm{O}_{3}(\mathrm{PZT})$ films has been investigated in view of their application in ultrasonic micro-actuators. The behaviour of resonance frequencies and quasistatic deflections has been studied as a function of membrane thickness and d.c. bias. The total stress in the films and the piezoelectric constant, $d_{31}$, have been derived by means of two different methods. The results are consistent with direct strain measurements by optical interferometry and with bulk ceramic values of identical composition.
\end{abstract}

Keywords: Piezoelectricity; Thin films; Ultrasonic actuators

\section{Introduction}

Thin diaphragms or membranes actuated by piezoelectric thin films are of interest for ultrasonic transducers and actuators. Standing deflection waves, travelling waves or Lamb waves have been explored for micromotors [1,2] and micro-pumps [3]. Acoustic applications have also been investigated [4]. The first demonstrations of such thin film devices have been realized with the piezoelectric material ZnO. In the past years much effort has been expended to integrate the ferroelectric material PZT $\left(\mathrm{PbZr}_{x} \mathrm{Ti}_{1-x} \mathrm{O}_{3}\right)$ on silicon devices. As known from bulk ceramics, PZT is one of the best piezoelectric materials found to date. Its relevant piezoelectric coefficients are an order of magnitude larger than those of $\mathrm{ZnO}$.

The first working PZT $\left(\mathrm{PbZr}_{x} \mathrm{Ti}_{1-x} \mathrm{O}_{3}\right)$ thin film micro motor has recently been demonstrated by the authors [5]. The application in micromotors is especially interesting since ultrasonic motors are considered to be superior to other types of micromotors for down scaling [6]. The motor was a hybrid type with an elastic fin rotor, proposed by Kurosawa et al. [7,8] for down scaling, and whose micromechanical version was first realized by Racine et al. [2] with $\mathrm{ZnO}$ thin films. As expected, the speed of PZT micromotors as compared to $\mathrm{ZnO}$ micromotors was higher at a given voltage. An improvement of a factor of 4 to 6 was found. The motor could be operated with voltages as low as $1.0 \mathrm{~V}_{\mathrm{rms}}$, which is sufficiently low for standard battery and IC supply voltages. Yield and reliability of the stator membranes turned out to be quite good and first degradation tests under operating conditions revealed a decrease in amplitude of $5 \%$ in $100 \mathrm{~h}$ [9].

In this work the membranes with no load are investigated in more detail. For design and simulation of the motor, or any other application, the behavior of the membrane should be able to be predicted. The unknown parameters are the total mechanical stress of all the constituent films of the membrane, and the relevant piezoelectric constant of PZT. It should be possible to extract these parameters from resonance frequency shifts and static deflection amplitudes as a function of diaphragm thickness and applied electric field. Finally, it is also of interest to relate the so obtained piezoelectric constant to the known bulk ceramic values. 


\section{Experimental}

\subsection{Fabrication of the diaphragms}

The piezoelectric thin film diaphragms were micro machined out of 3" silicon wafers. Apart from the remaining silicon, the diaphragms consisted of $\mathrm{SiO}_{2}, \mathrm{Si}_{3} \mathrm{~N}_{4}$, electrode and PZT films. The structure was made as simple as possible and is shown schematically in Fig. 1. The electrode below the PZT film (bottom electrode of $\mathrm{Pt} / \mathrm{Ta}$ ) was not structured. Contact pads, conductor lines and top electrodes were patterned from the same aluminum film, deposited directly on the PZT layer. Details of the fabrication have been reported elsewhere [5]. The sputter deposited PZT film of the present study had a composition of 45/55, (100) texture, a dielectric constant of 870 , and a dielectric loss of $4 \%$ (at $1 \mathrm{kHz}$ and $100 \mathrm{mV}$ ). It was grown on a $30 \mathrm{~nm} \mathrm{PbTiO}_{3}$ template at about $600^{\circ} \mathrm{C}[10]$.

Three sizes of circular membranes with diameters of 2, 4 and $8 \mathrm{~mm}$ have been fabricated. Top electrodes of half the diameter of the membrane allowed excitation of the ground mode $\mathrm{B}_{00}$ as well as the $\mathrm{B}_{10}$ mode with one circular node.

\subsection{Deflection measurements by optical interferometry}

The deflections of the membranes have been analyzed by means of a Michelson interferometer [5,11] working with a He-Ne laser. In order to measure the deflection in the linear range of the interferometer, the ac excitation voltage was only a few $\mathrm{mV}$. The resonance spectrum was measured by scanning through the frequencies between 1 and $100 \mathrm{kHz}$ [5]. Resonance amplitudes of up to $1.1 \mu \mathrm{m} / \mathrm{V}$ have been found (ground mode $\left(\mathrm{B}_{00}\right)$ at $53 \mathrm{kHz}$ with a $16 \mu \mathrm{m}$ thick, $2 \mathrm{~mm}$ wide membrane covered with $\left.0.6 \mu \mathrm{m} \mathrm{PZT}\right)$. The symmetry of the modes has been determined by mapping the nodal lines with quartz powder (Chladni figures).

For not too small thicknesses, the membranes are in principle well approximated by the model for thin clamped circular disks, for which exact values of the resonance frequencies are available [12]. In the limit of zero thickness, pure membrane behavior should be observed. Also in this case the resonance frequencies are tabulated [12]. In order to study the intermediate behavior and to quantify the film stress contribution, the spectrum of the same $2 \mathrm{~mm}$ membrane was measured for different silicon thicknesses. After each measurement the silicon was thinned down further by back side etching in $\mathrm{KOH}$. A crossover from membrane to disk behavior could indeed be observed (see Fig. 2), that is, the $(h)^{-1 / 2}$ slope for a small silicon thickness, $h$, changes to a slope proportional to $h$ for greater thicknesses. The diaphragm is obviously under a small tension, leading to the frequency increase at small thicknesses. The curve fitting is explained in section 3 .

When a dc field $\left(E_{3}\right)$ parallel to the polarization (i.e. $\left.E_{3}>0\right)$ is added to the ac field, the resonance frequency is shifted to higher frequencies. The effective piezoelectric stress coefficient $\tilde{e}_{31}$ is different from the $e_{i k}=-\partial \sigma_{k} / \partial E_{i}$ of the fully clamped sample, as is shown in the appendix. The in-plane piezoelectric stress, now written as $\sigma_{p}=-\tilde{e}_{31} \cdot E_{3}$, which is tensile in this case, adds to the stretching forces of the film stresses. For anti parallel fields (smaller than the coercive field) the resonance frequency decreases. Due to the hysteresis of polarization a "butterfly" figure is obtained (see Fig. 3). As a small perturbation, the piezoelectric stress shifts the resonance frequency in a linear manner: 

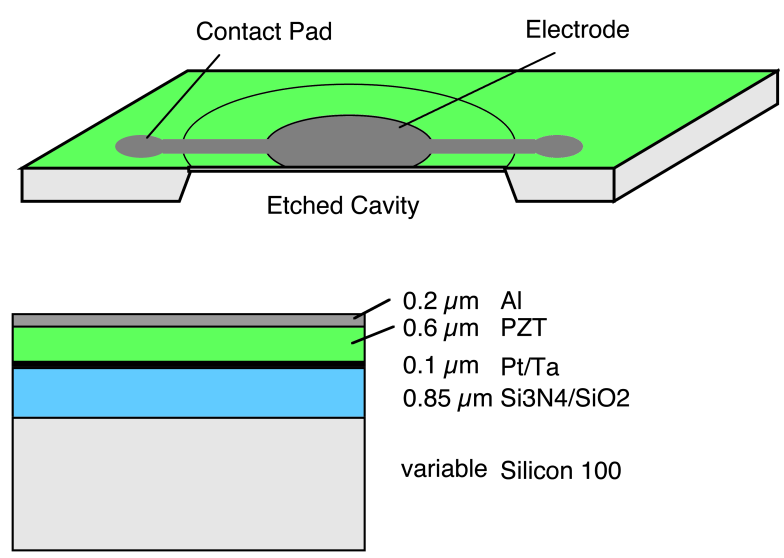

Figure 1. Schematic drawing of the membrane with view onto the top side of the wafer and a schematic cross section through the films.

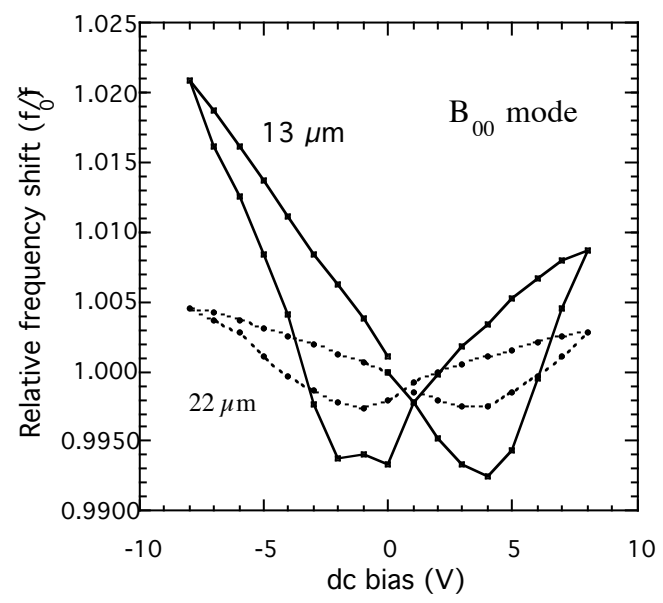

Figure 3. Frequency vs. de bias loops for two thicknesses of $2 \mathrm{~mm}$ diameter diaphragms with sputtered $0.6 \mu \mathrm{m}$ thick PZT films. The center frequencies are 42.3 and $71.6 \mathrm{kHz}$ for the 13 and $22 \mu \mathrm{m}$ membranes respectively. The same samples as for Fig. 2 have been used.

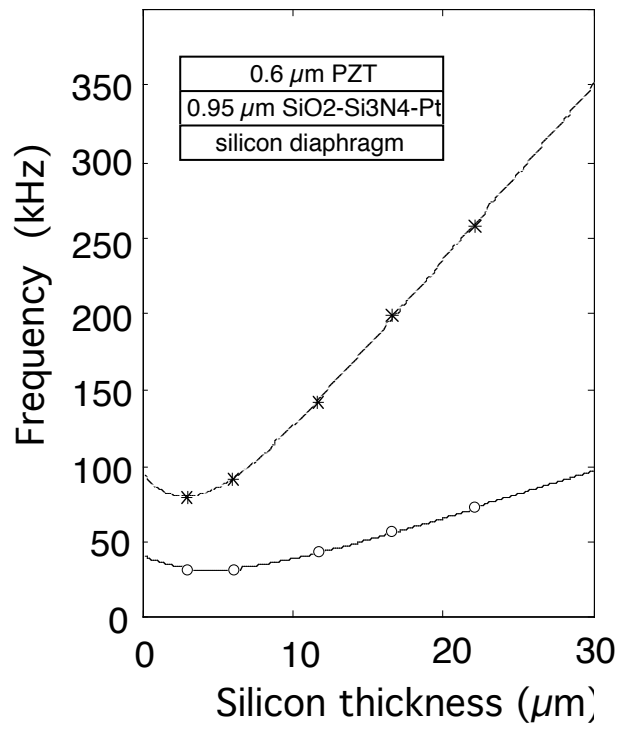

Figure 2. Resonance frequencies as a function of the silicon thickness of $2 \mathbf{~ m m}$ diameter diaphragms with sputtered PZT films. The curve fit is discussed in the text.

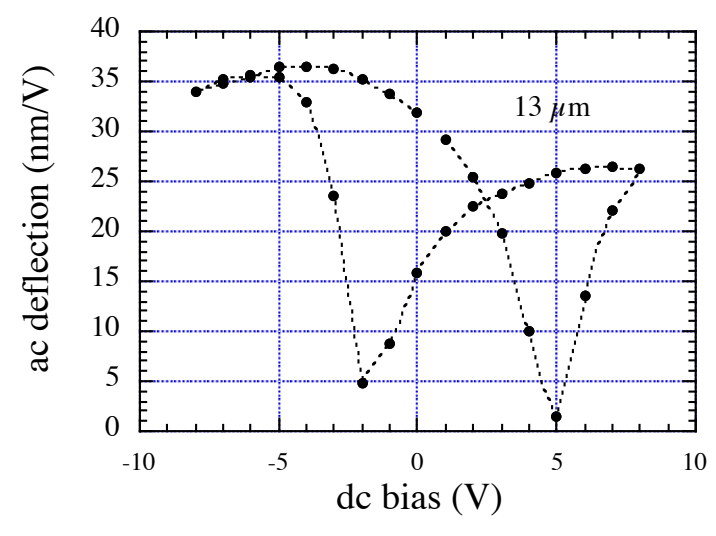

Figure 4. Interferometric measurements of membrane deflections as a function of de bias applied to the $0.6 \mu \mathrm{m}$ thick sputtered PZT film. Sample as used in Figs 2 and 3. 


$$
\Delta \omega \propto-\tilde{e}_{31}\left(E_{3}\right) \cdot E_{3}
$$

Note that the effective piezoelectric coefficient $\tilde{e}_{31}$ is negative. The derivation of the proportionality factor is explained in section 3. The experiment was performed with two thicknesses of the same sample as used for the stress determination described above.

An alternative way to determine the piezoelectric constant consists in calculating the static deflection. The latter is reduced by the tensile stresses in the films. However, these are known from the first experiment and can be taken into account. Fig. 4 shows the deflection as a function of dc bias at $1 \mathrm{kHz}$ for the $13 \mu \mathrm{m}$ sample, normalized for a voltage of $1 \mathrm{~V}$. This result should be very close to the static response, since there is no longer any dispersion at this low frequency. The static response is proportional to the effective piezoelectric coefficient $\tilde{e}_{31}$ :

$$
\frac{\text { deflection amplitude }}{\text { ac voltage amplitude }} \propto\left|\tilde{e}_{31}\left(E_{3}\right)\right|
$$

Finally, a comparison with the piezoelectric strain $\varepsilon_{3}=\tilde{d}_{33} \cdot E_{3}$ perpendicular to the substrate plane has been made. This strain and the piezoelectric stress $\sigma_{p}$ in the plane should exhibit a similar behaviour as a function of the applied field. Fig. 5 shows that this is indeed the case. $\varepsilon_{3}$ was determined by a double beam interferometer, which measures the thickness change of the film on a standard $0.5 \mathrm{~mm}$ thick silicon substrate $[11,13]$.

\section{Theory and calculations}

In this section analytical calculations are presented. They allow a derivation of the static membrane deflection and the resonance frequencies as a function of size, film thicknesses, applied electric field and film properties. The film stresses have a strong influence on deflection and resonance frequencies. It is clear that the silicon part has no stress, since it is part of the single crystal substrate. In order to derive the piezoelectric constant from deflection data it is necessary to study and measure the stress contribution from the thin films on top of the silicon.

\subsection{The eigenfrequencies of stretched thin clamped disks}

It is assumed that the membranes are perfectly circular (radius $a$ ), that the silicon thickness $(h)$ and the film thicknesses $\left(t_{\mathrm{fi}}\right)$ are homogeneous and that the clamping of the diaphragm is perfect for all thicknesses considered. The deflection $w(r, t)$ of the diaphragm out of the wafer plane is described by $w(r, t)=F(r) \cdot \sin \omega t$, where $r$ is the distance from the center of the diaphragm and $\omega$ is the excitation frequency. It is further assumed, that the deflection is smaller than the thickness of the diaphragm. Nonlinear effects are thus neglected. The obvious boundary condition $F(a)=0$ must hold. 


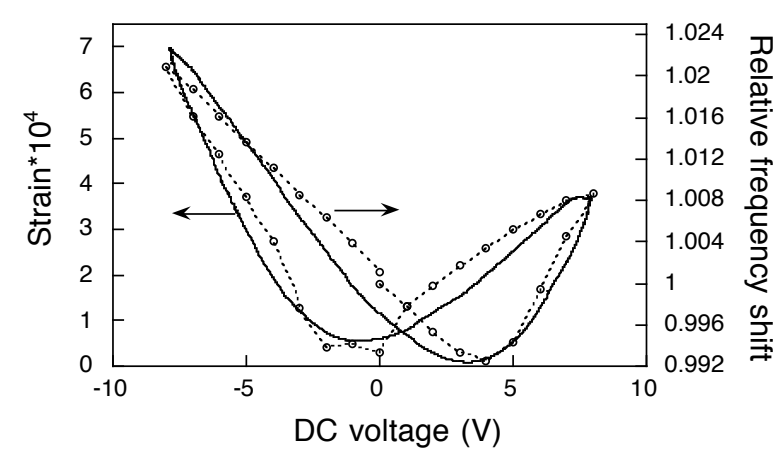

Figure 5. Double interferometric measurement of the film strain perpendicular to the substrate plane, compared with the stress sensing shift of the resonance frequency (13 $\mu \mathrm{m}$ diaphragm of Fig. 3).

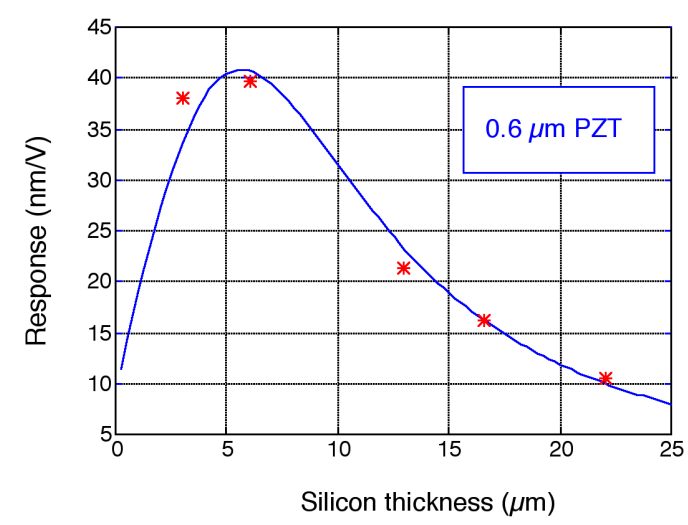

Figure 6. Calculated amplitude of the static deflection according to Eq. 15 for the case of the response of an unpoled film at zero dc bias and

$5 \mathrm{mV}$ ac electric excitation at $1 \mathrm{kHz}$. The experimental points have been obtained by a series of etching steps at the same device.

For the resonance behavior one can distinguish two limiting cases:

(1) Disk approximation: The diaphragm is rather thick and the stresses can be neglected. This is the well known thin clamped disk situation, which has the additional boundary condition:

$$
\left.\frac{d F}{d r}\right|_{r=a}=0
$$

The eigenfrequencies are determined by the elastic energy term $U_{\mathrm{el}}[12]$ :

$$
U_{e l}=\frac{1}{2} D \int_{0}^{a} 2 \pi r d r \times\left[\left(\frac{d^{2} F}{d r^{2}}+\frac{1}{r} \frac{d F}{d r}\right)^{2}-2(1-v) \frac{1}{r} \frac{d F}{d r} \frac{d^{2} F}{d r^{2}}\right]
$$

where

$$
D=\frac{Y \cdot h^{3}}{12\left(1-v^{2}\right)}
$$

$D$ is given for a single layer diaphragm with the Young's modulus and $v$ the Poisson ratio. The solutions $F D_{\mathrm{k}}(r)$ of the $k^{\text {th }}$ resonance are known [12]:

$$
F D_{k}(r)=c_{1} \cdot J_{0}\left(\lambda_{k} \cdot \frac{r}{a}\right)+c_{2} \cdot J_{0}\left(i \lambda_{k} \cdot \frac{r}{a}\right)
$$

$J_{0}$ is the Bessel function of order 0 . For the first two modes $\mathrm{B}_{00}$ and $\mathrm{B}_{10}$ (adopting the usual notation with the first index being the number of circular nodes) $c_{2} / c_{1}$ amounts to 0.056 and -0.025 respectively. The coefficients $\lambda_{\mathrm{k}}$ are 3.190 and 6.306, respectively [12]. The eigenfrequencies are obtained as [12]: 


$$
\omega_{k}^{2}=\frac{\lambda_{k}^{4}}{a^{4}} \cdot\left\{\frac{D}{\mu}\right\}
$$

where $\mu$ is the mass density per unit area of the diaphragm. It follows that the resonance frequencies increase linearly with the thickness of the diaphragm.

(2) Membrane approximation: The diaphragm is thin, and the stretching forces caused by film stresses are dominating. The stretching force per unit length $S$ is acting on the border of the membrane in radial direction. The potential energy is in this case:

$$
U_{S}=\frac{1}{2} S \int_{0}^{a} 2 \pi r d r \cdot\left(\frac{d F}{d r}\right)^{2}, \quad S=S_{f}+S_{p}, \quad S_{f}=\sum \sigma_{f i} \cdot t_{f i}
$$

$S_{\mathrm{f}}$ originates from film stresses, $S_{\mathrm{p}}$ from the piezoelectric effect. $\sigma_{\mathrm{fi}}$ is the stress in the $i^{\text {th }}$ thin film of thickness $t_{\mathrm{fi}}$. The solutions $F M_{\mathrm{k}}(r)$ at resonance are simply the Bessel functions $J_{0}$ with zero values at $\mathrm{r}=\mathrm{a}$ :

$$
F M_{k}(r)=c \cdot J_{0}\left(x_{0 k} \cdot \frac{r}{a}\right)
$$

For $\mathrm{B}_{00}$ and $\mathrm{B}_{10}$ the $x_{0 \mathrm{k}}$ amount to 2.405 and 5.52. The eigenfrequencies are then obtained as:

$$
\omega_{k}^{2}=\frac{x_{0 k}^{2}}{a^{2}} \cdot\left\{\frac{S}{\mu}\right\}
$$

In this case the eigenfrequencies drop with increasing the membrane thickness as $(h)^{-1 / 2}$.

In order to obtain an expression for the intermediate case, the Rayleigh-Ritz method was applied. The kinetic energy term is written as:

$$
E_{k i n, \max }=\omega^{2} \cdot I_{k i n} \quad I_{k i n}=\frac{\mu}{2} \int_{0}^{a} 2 \pi r F^{2} d r
$$

At resonance the maximum kinetic energy is equal to the maximum potential energy and one arrives at:

$$
\omega_{k}^{2}=\frac{U_{e l}\left(F_{k}\right)}{I_{k i n}\left(F_{k}\right)}+\frac{U_{S}\left(F_{k}\right)}{I_{k i n}\left(F_{k}\right)}
$$

The exact solution $F_{\mathrm{k}}$ yields the exact resonance frequency. With an approximate solution, respecting the symmetry of the mode $k$, an approximate value is obtained.

Taking $F D$ as the deflection function one obtains good values for $\omega_{\mathrm{k}}$ in the case where $D / a^{2}>>\mathrm{S}$. This was done for calculating the frequency shift by the piezoelectric stress (Fig. 3). The parameters $D$ and $\mu$ have been calculated for the three layer situation with (1) silicon, (2) $\mathrm{SiO}_{2}-\mathrm{Si}_{3} \mathrm{~N}_{4}-\mathrm{Pt}$ and (3) PZT. The formulas for $D$ in the multi layer situation are given in Ref. [16].

For covering the whole range of thicknesses a further approximation was made. The functions $F D$ and $F M$ are rather similar, and therefore they also yield about the same values for the integrals $U_{\mathrm{el}}, U_{\mathrm{S}}$ and $E_{\text {kin }}\left(\mathrm{U}_{\mathrm{S}}\right.$ $(F D)$, for instance, differs indeed only by $10 \%$ from $U_{\mathrm{S}}(F M)$ ). The left side of the sum in E. 8 is evaluated with $F D$, the right side with $F M$. The following is thus obtained: 


$$
\omega_{k}^{2} \approx \frac{\lambda_{k}^{4}}{a^{4}} \cdot\left\{\frac{D}{\mu}\right\}+\frac{x_{k}^{2}}{a^{2}} \cdot \frac{S}{\mu}
$$

which is exact for very thin $(D=0)$ and for very thick diaphragms $\left(D / a^{2}>>S\right)$. For the curve fit in Fig. 2 the multilayer values for $D$ and $\mu$ have been applied. The only free parameter was $S$. For a perfect fit , though, $\lambda_{00}$ had to be slightly increased by $3 \%$, probably due to inaccurate geometry.

\subsection{The piezoelectric energy terms}

The piezoelectric stress $\sigma_{\mathrm{p}}$ in the PZT film acts in two ways on the diaphragm. First, it creates a bending moment with respect to the neutral plane of the diaphragm. Second, it contributes to the stretching forces $S$. The bending moments cause the deflection of the diaphragm, the stretching effect shifts the resonance frequency. It is therefore possible to deduce the piezoelectric constant from the deflection and the frequency shift.

The piezoelectric film is clamped to the substrate (the diaphragm in this case) in the film plane (directions with indices 1,2 ) and is free to move perpendicular to it (index 3), i.e. $\sigma_{3}=0$. The stresses in the plane, $\sigma_{1}$ and $\sigma_{2}$, are equal by symmetry and obtained as (see appendix):

$$
\sigma_{p}\left(E_{3}\right)=\sigma_{1}\left(E_{3}\right)=\sigma_{2}\left(E_{3}\right)=-\tilde{e}_{31} \cdot E_{3}
$$

where

$$
\tilde{e}_{31}=\frac{d_{31}}{s_{11}^{E}+s_{12}^{E}}=\left(e_{31}+\frac{s_{13}^{E} \cdot e_{33}}{s_{11}^{E}+s_{12}^{E}}\right)
$$

Note that the effective constant $\tilde{e}_{31}$ is larger than $e_{31}$, which is defined for full clamping. This compensates the fact that the effective $\tilde{d}_{33}$ is smaller than the $d_{33}$ of the free bulk sample [14]. Since $\tilde{e}_{31}$ is negative, one obtains a tensile stress if $E_{3}$ is positive, i.e. parallel to the polarization. The stretching force imposed by the piezoelectric stress is: $S_{\mathrm{p}}=\sigma_{\mathrm{p}} \cdot t_{\mathrm{p}} / 2$ ( $t_{\mathrm{p}}$ is the PZT film thickness). The factor $1 / 2$ originates from the fact that the electrode has only half the circumference of the membrane. The bending moment is obtained as:

$$
M_{x}=M_{y}=M=\sigma_{p}\left(E_{3}\right) \cdot t_{p} \cdot \frac{h_{e f f}}{2}
$$

where $h_{\text {eff }} / 2$ is the distance between the center of the piezoelectric film and the neutral plane of the diaphragm, and was calculated according to Ref. [16]. In the case of the measured $13 \mu \mathrm{m}$ membrane (Fig. 4) $h_{\text {eff }}$ is close to $h$. The potential energy due to $M$ is given by [15]:

$$
U_{M}=\frac{1}{2} M \int_{0}^{a / 2} 2 \pi r d r \cdot\left(\frac{d^{2} w}{d r^{2}}+\frac{1}{r} \frac{d w}{d r}\right)
$$

The integral covers the area of the electrode only.

\subsection{The static deflection}

The static problem was solved by the so called energy method, i.e. by minimizing the potential energy with suitable test functions [15]. The energy consists of three parts: $U_{\text {el }}$ is the elastic energy due to the bending of the disk, $U_{\mathrm{M}}$ is the work done by the piezoelectric moment $M$, and $U_{\mathrm{S}}$ is the stretching energy due to the (tensile) film stresses. The following function was taken as a test function: 


$$
w(r)=\left\{\begin{array}{c}
\gamma \cdot\left(2 \ln 2 \cdot a^{2}-3 \cdot r^{2}\right) \\
\gamma\left(r^{2}-a^{2}-2 \cdot a^{2} \cdot \ln (r / a)\right.
\end{array}\right\} \begin{array}{r}
, r \leq \frac{a}{2} \\
, r \geq \frac{a}{2}
\end{array}
$$

The two parts obey the differential equation for disks with zero lateral forces $(\Delta \Delta w=0)$, have a constant $\Delta w$, and have the correct boundary conditions for clamping at $r=a$. The minimum condition $\mathrm{d} U / \mathrm{d} \gamma=0$ yields the optimal value for $\gamma$ and the deflection in the center $w(0)$ is obtained:

$$
w(0)=\frac{3 \ln (2) \cdot a^{2} \cdot M}{2.55 a^{2} \cdot S+48 D}
$$

According to this equation a maximum deflection is expected at an intermediate thickness, depending on the stress level. It was indeed observed that the piezoelectric response first increased and then diminished with reducing the silicon thickness (see Fig. 6). The film in this experiment was not poled. Piezoelectricity is due to self poling during the fabrication process. The calculated amplitude $w(0)$ displayed in Fig. 6 was obtained with the stress parameter $S$ derived from Fig. 2 and with a piezoelectric coefficient $\tilde{e}_{31}$ of $2.6 \mathrm{C} / \mathrm{m}^{2}$. It turns out that the maximum of deflection occurs at about the same diaphragm thickness where the resonance frequency has its minimum (see Fig. 2).

\section{Results and discussion}

The curve fitting in Fig. 2 yielded an average film stress of $80 \mathrm{MPa}$ (tensile), which is a rather low value. The $\mathrm{Si}_{3} \mathrm{~N}_{4} / \mathrm{SiO}_{2}$ part was indeed designed to have a low stress of $100 \mathrm{MPa}$ (tensile) for pyroelectric applications, where the silicon is completely etched away [17]. The contribution of platinum and PZT is thus also tensile and amounts to about $60 \mathrm{MPa}$.

The effective piezoelectric coefficient $\tilde{e}_{31}$ has been derived from the resonant method (Fig. 3) and the static deflection method (Fig. 4). The first yielded values of $5.0 \mathrm{C} / \mathrm{m}^{2}(13 \mu \mathrm{m})$ and $4.7 \mathrm{C} / \mathrm{m}^{2}(22 \mu \mathrm{m})$, the latter yielded values of $3.7 \mathrm{C} / \mathrm{m}^{2}$ at zero field and $4.2 \mathrm{C} / \mathrm{m}^{2}$ at saturation for the $13 \mu \mathrm{m}$ diaphragm. Without taking into account the film stress, $20 \%$ smaller values would have been obtained. A larger membrane with $4 \mathrm{~mm}$ diameter and $20 \mu \mathrm{m}$ thickness, with the same sputtered film, was also evaluated for its static deflections. The poled film yielded a $\tilde{e}_{31}$ of $4.1 \mathrm{C} / \mathrm{m}^{2}$ at zero field. The agreement between different membranes and thicknesses, and between the two methods is thus satisfying.

How do these values compare with PZT bulk ceramics? Unfortunately no values are available for the composition $45 / 55$ of $\mathrm{Zr} / \mathrm{Ti}$. $\tilde{e}_{31}$ decreases from $9.6 \mathrm{C} / \mathrm{m}^{2}$ at $52 / 48$ composition, which shows the largest piezoelectric activity, to $5.8 \mathrm{C} / \mathrm{m}^{2}$ at $48 / 52$ composition [18]. Extrapolating these data, the obtained $\tilde{e}_{31}$ values seem to be reasonable. In spite of using PZT of non optimized composition, the relevant piezoelectric coefficient is 4 times higher than in $\mathrm{ZnO}$ with a calculated $\tilde{e}_{31}$ of $1.1 \mathrm{C} / \mathrm{m}^{2}$ (single crystal data: $d_{31}=-5 \mathrm{pm} / \mathrm{V}, s_{11}=7.9 \cdot 10^{-12} \mathrm{~Pa}^{-1}$ and $\left.s_{12}=-3.4 \cdot 10^{-12} \mathrm{~Pa}^{-1}[19]\right)$.

Since usually the $d_{\mathrm{ij}}$ coefficients are given as material parameters, it makes sense to derive here also $d_{31}$, taking tabulated bulk ceramic values for the compliance coefficients $\left(s_{11}=10.8 \cdot 10^{-12} \mathrm{~Pa}^{-1}\right.$ and $s_{12}=$ $3.35 \cdot 10^{-12} \mathrm{~Pa}^{-1}$ for PZT 48/52 [18]). One has to keep in mind, however, that the values given below are only as precise as the elastic constants used for their derivation. The so obtained $d_{31}$ values scatter between 31 and $37 \mathrm{pm} / \mathrm{V}$ in saturation. These values seem to be correct for the $45 / 55$ composition $\left(d_{31}=43 \mathrm{pm} / \mathrm{V}\right.$ for 
PZT 48/52 [18]). One would expect that the ratio $d_{33} / d_{31}$, which amounts to 0.40 in PZT 50/50 ceramics, would apply also for thin films. The effective piezoelectric coefficient $\tilde{d}_{33}$ was derived from the slope of $\varepsilon_{3}$ vs. $E_{3}$ in Fig. 5 and obtained as $49 \mathrm{pm} / \mathrm{V}$. Again, the measured $\tilde{d}_{33}$ is not equal to the coefficient $d_{33}$ of the free sample due to clamping effects. The correction is given in ref.14 and is calculated as $\tilde{d}_{33} / d_{33}=0.66$, applying elastic compliance coefficients of bulk ceramic PZT $48 / 52$. With the corrected $d_{33}$ of $74 \mathrm{pm} / \mathrm{V}$ in saturation, a value 0.42 to 0.50 is obtained for $d_{33} / d_{31}$ in the thin film.

The best operating conditions can be derived from Figs 3 and 4. Obviously, the PZT films applied had a built-in asymmetry: the poling with negative top electrode yielded a much larger piezoelectric response than the poling in opposite direction. After fabrication, the films were already polarized to about $60 \%$ of their response achieved by poling at room temperature. The best operating conditions were found to lie between -8 and $+2 \mathrm{~V}$, where polarization switching was avoided. This asymmetry might be helpful, since it enables a larger piezoelectric (and also pyroelectric) response at low fields, i.e. the remanence is improved for one polarization direction.

For an optimal application of the membrane as a vibrating stator, an ideal relationship between the thickness of PZT film and the thickness of the silicon part has to be found. Equation 15 gives an approximate solution for the case of an unloaded membrane out of resonance without air damping or other forces. It shows that the film stresses are of importance and shift the ideal silicon thickness to larger values. Analogously, external forces, such as the load of a rotor, are expected to shift the optimal thickness to higher values.

\section{Conclusions}

PZT activated membranes could be characterized with respect to resonance frequencies, total thin film stress, and piezoelectric coefficients. The behavior was analyzed by means of analytical calculations. It turned out that film stress needs to be taken into account for optimal design of the membrane. The different experiments show consistent results of the derived piezoelectric coefficients, which seem to agree with bulk ceramics data. For precise comparison, however, the exact values of the elastic compliance tensor would be required. The PZT films showed an effective stress piezoelectric coefficient which was a factor four larger than that for $\mathrm{ZnO}$ single crystals. Optimization of film composition still may increase this coefficient by another factor two.

\section{Acknowledgments}

This work was supported by the Materials Priority Program of the Board of the Swiss Federal Institutes of Technology, the Swiss Federal Office of Education and Science (OFES), and the European COST 514 program. 


\section{Appendix}

The stress components $\sigma_{1}$ and $\sigma_{2}$ are in the plane of the diaphragm, $\sigma_{3}$ out of plane. The same notation is given to the strain components $\varepsilon_{1}, \varepsilon_{2}, \varepsilon_{3}$. Since the film is free to move in the 3-direction the stress component $\sigma_{3}$ must be zero.

Equations of state:

$$
\begin{aligned}
& \varepsilon_{1}=s_{11}^{E} \sigma_{1}+s_{12}^{E} \sigma_{2}+d_{31} E_{3} \\
& \varepsilon_{2}=s_{12}^{E} \sigma_{1}+s_{11}^{E} \sigma_{2}+d_{31} E_{3}
\end{aligned}
$$

The sum of these two equations yields the average in plane stress:

$$
\frac{\left(\sigma_{1}+\sigma_{2}\right)}{2}=\frac{\varepsilon_{1}+\varepsilon_{2}-2 d_{31} \cdot E_{3}}{2\left(s_{11}+s_{12}\right)}
$$

Directions 1 and 2 are equivalent, since the PZT film is isotropic in the plane, and since membranes and electrodes have cylindrical symmetry. With perfect clamping (i.e. no strain in the plane due to piezoelectricity) the final result becomes:

$$
\sigma_{1}=-\frac{d_{31} \cdot E_{3}}{s_{11}+s_{12}}=-\tilde{e}_{31} \cdot E_{3}
$$

\section{References}

[1] K.R. Udayakumar, S.F. Bart, A.M. Flynn, J. Chen, L.S. Tavrow, L.E. Cross, R.A. Brooks, and D.J. Ehrlich, Ferroelectric thin film ultrasonic micromotors, Proc. IEEE-MEMS, Nara, Japan, 1991, pp.109-113.

[2] G.-A. Racine, R. Luthier, and N.F. de Rooij, Hybrid ultrasonic micromachined motors, Proc. IEEE-MEMS 93 (Fort Lauderdale, USA, 1993) pp. 128-132.

[3] R.M. Moroney, R.M White, and R.T. Howe, Ultrasonic micromotors: physics and applications, Proc. IEEEMEMS, Napa Valley, USA, 1990, pp. 182-187.

[4] See, e.g. P.R. Scheeper, A.G.H. van der Donk, W. Olthuis, and P. Bergveld, A review of silicon microphones, Sensors and Actuators A, 44 (1994) 1-11.

[5] P. Muralt, M. Kohli, T. Maeder, A. Kholkin, K. Brooks, R. Luthier, and N. Setter, Fabrication and characterization of PZT thin film vibrators for micromotors, Actuators and Sensors 48 (1995) 157-165.

[6] A.M. Flynn, L.S. Tavrow, S.F. Bart, R.A. Brooks, D.J. Ehrlich, K.R. Udayakumar, and L.E. Cross, Piezoelectric micromotors for microrobots, J. Microelectromechanical Systems, 1 (1992) 44-51.

[7] T. Uchiki, T. Nakazawa, K. Nakamura, M. Kurosawa, and S. Ueha, Ultrasonic motor utilizing elastic fin rotor, Jap. J. Appl. Phys. 30 (1991) 2289-2291.

[8] M. Kurosawa, T. Uchiki, H. Hanada, K. Nakamura, and S. Ueha, Simulation and experimental study on elastic fin ultrasonic motor, IEEE Ultrasonics Symposium 92 (Tucson, USA, 1992) pp. 893-896.

[9] P. Muralt, A. Kholkin, M. Kohli, T. Maeder, K. Brooks, and R. Luthier, Fabrication and characterization of PZT thin films for micromotors, ISIF'95, Colorado Springs, USA, J. Int.Ferroelectrics 11 (1995) 213-220.

[10] T. Maeder, P. Muralt, L. Sagalowicz, and N. Setter, In-situ sputter deposition of PT and PZT films on Platinum and $\mathrm{RuO}_{2}$ electrodes, 1st Eur. Meet. Integrated Ferroelectrics, Nijmegen, Netherlands, 1995, J. Microelectronic Engineering 29 (1995) 177-180.

[11] A. Kholkin, E. Colla, K. Brooks, P. Muralt, M. Kohli, T. Maeder, D. Taylor, and N. Setter, Interferometric study of piezoelectric degradation in ferroelectric thin films, 1st Eur. Meet. Integrated Ferroelectrics, Nijmegen, Netherlands, 1995, J. Microelectronic Engineering 29 (1995) 261-264.

[12] Istvan Szabo, Höhere Technische Mechanik, Springer, Berlin, 1956.

[13] A. Kholkin, Ch. Wüthrich, D. Taylor, and N. Setter, Interferometric Measurements of electric field-induced displacements in piezoelectric thin films, Rev. Sc.Instr., submitted.

[14] K. Lefki and G.J.M. Dormans, Measurement of piezoelectric coefficients of ferroelectric thin films, J.Appl.Phys. 76 (1994) 1764-1767. 
[15] S. Timoshenko, S. Woinowsky-Krieger, Theory of Plates and Shells, McGraw-Hill, New York, 1959.

[16] J. Söderkvist, Similarities between piezoelectric, thermal and other internal means of exciting vibrations, J. Micromech. Microeng. 3 (1993) 24-31.

[17] A. Bell, Y. Huang, M. Kohli, O. Paul, P. Ryser, and M. Forster, $\mathrm{PbTiO}_{3}$ thin films for pyroelectric detectors, Proc. Int. Symp. Appl. Ferroelectrics (ISAF), Pennsylvania, USA, 1994, pp. 691-694.

[18] Landolt and Bernstein, New series III/16a, Ferroelectrics: Oxides, Springer, New York, 1981.

[19] T. Ikeda, Fundamentals of Piezoelectricity, Oxford University Press, Oxford, 1990.

\section{Biographies}

Paul Muralt received his diploma in physics and the Ph.D. degree for his investigations of incommensurate structural phase transitions from the Swiss Federal Institute of Technology in Zurich. In 1984 and 1985 he held a post-doctoral position at the IBM Research Laboratory in Zurich, working in the field of tunnelling microscopy and spectroscopy. Next, he started new activities in tunnelling microscopy at the Free University of Berlin. In 1987 he switched to industry, joining the Balzers group in Liechtenstein. He became a specialist in sputter-deposition techniques and managed a department for PVD process development and application. In 1993 he returned to university and joined the laboratory of ceramic materials at the Swiss Federal Institute of Technology in Lausanne. His research interests are the deposition of ferroelectric thin films and the application of piezo- and pyroelectric thin films in micro sensors and actuators.

Andrei Kholkin studied physics at the St. Petersburg State University and in 1988 received the Ph.D. at the Joffe Institute in St. Petersburg for his work on transport properties and kinetic phenomena in ferroelectrics. Afterwards, he continued at the same institute and extended his research to high- $T_{\mathrm{C}}$ superconductors. In 1994 he joined the laboratory of ceramic materials at the Swiss Federal Institute of Technology in Lausanne. His research interests are now the characterization of piezoelectric thin films and membranes.

Markus Kohli was born in 1967. He studied physics at the Swiss Federal Institute of Technology in Zurich and received his diploma in 1993. He is now working on his Ph.D. thesis on the subject of materials and processing for micromechanics.

Thomas Maeder was born in 1970. He studied material sciences at the Swiss Federal Institute of Technology in Lausanne and received his diploma in 1992. He is now working on his Ph.D. thesis on fabrication and characterization of piezoelectric thin films. 